\title{
Effectiveness of Modified Hybrid Brainstorming Learning Program in Increasing Innovative Work Behavior and Innovative Idea Performance among Nurses
}

\author{
Katekanok Kamonmarttayakul ${ }^{1, *}$, Nongnut Boonyoung ${ }^{2}$ and Sasitorn Phumdoung ${ }^{2}$ \\ ${ }^{1}$ Quality Center, Bangkok Hospital Phuket, Phuket 83000, Thailand \\ ${ }^{2}$ Faculty of Nursing, Prince of Songkla University, Songkhla 90112, Thailand
}

("Corresponding author's e-mail: tkatekanok@gmail.com)

Received: 14 July 2020, Revised: 6 May 2021, Accepted: 14 May 2021

\begin{abstract}
The ability to learn and innovate has been known as a key-factor for nurses to deliver quality of care and patient safety. To be successful in nursing innovation, nurses should perform sound innovative behaviors; thus, their innovative work behavior (IWB) should be promoted. A comprehensive learning program aimed at improving IWB with effective cognitive stimulation could enhance innovation abilities and quality of innovative idea performance. This study aimed to evaluate the effectiveness of a learning program that uses Modified Hybrid Brainstorming (MHB) techniques in increasing IWB and improving the quality of ideas generated (fluency, flexibility and originality) among nurses. A sample of 60 nurses were randomly assigned to a within group pre-test and post-test study design. The learning program with the MHB method was developed and implemented for 3 sessions workshop. IWB was measured pre- and 12 weeks post-program. Pre- and post-training session fluency, flexibility, and originality of generated ideas were also measured. The percentage of absolute agreement and the index of item- objective congruence of research instruments were met. Data were analyzed using descriptive statistics, and paired t-test. The results revealed that the average IWB scores of nurses after the implementation of the MHB learning program increased significantly $(p<0.05)$. There was also a significant difference in post-test scores $(p<0.05)$ of fluency, flexibility, and originality of ideas. This study showed that offering the MHB learning program to nurses is a feasible method to improve IWB and innovative idea.
\end{abstract}

Keywords: Flexibility, Fluency, Innovative work behavior, Modified hybrid brainstorming learning program, Originality

\section{Introduction}

Nurses are a vital member of healthcare teams. With rising healthcare demands, nurse shortages, and resources limitations, nurses could be at the forepart of innovation to improve quality and safety of patient care [1]. To promote nursing innovation, nursing organizations should be searching to include learning programs that foster nurses' capability to innovate [2]. Innovative work behavior (IWB) is a crucial manner in generating innovation [3]. Additionally, previous studies have shown that organizational learning program regarding experiential learning theory (ELT) have a significant influence on the formation of nurses' innovative work behaviour [4,5].

IWB is a set of behaviors; idea exploration, idea generation, idea championing and implementation of ideas [6], which directly impact to innovation development [5]. The idea exploration focuses on clearly recognize a work problem, or opportunity that may be improved by innovation [7,8]. For this intent, critical thinking and effective systematic tools are needed and used [9]. Generally, conventional brainstorming (CB) [10] applying the 5W1H questions (What, Where, When, Why, Who and How), is used to address well-defined statement of problems [11].

The idea generation is a manner to generate new ideas which may relate product, process, service or technology [12]. The important factors that enhance idea generation manner are creativity (divergent and convergent thinking) [13,14], effective cognitive stimulating methods [15], appropriate group structure and group interaction styles [15,16], and the suitable external stimuli [17]. Additionally, previous studies have revealed that brainstorming and analogical thinking are effective methods to promote learner's cognition and enhance idea generation $[15,18]$. Substantiating, studies showed that brainstorming is one 
of the cognitive stimulating methods that promotes group creativity $[15,19]$. Brainstorming enhances all idea generation under the 4 basic rules: 1) to generate as many ideas as possible; 2) no one is to criticize an idea; 3) to produce wild ideas; and 4) to combine ideas or improve on each other's ideas [20].

Generally, brainstorming methods are used in 2 techniques; face-to-face technique (conventional brainstorming: $\mathrm{CB}$ ) [19] and paper-pencil technique (brainwriting: $\mathrm{BWr}$ ) [15]. CB is the first form of group-based brainstorming, in which group members actively participate in dialogue and interact by sharing their thoughts throughout the session [19]. Even though CB is useful for both idea exploration and idea generation manners, its negative effects include production blocking or inhibition of other members from offering ideas during discussion, apprehension of evaluation, and social loafing or free riding [21]. Thus, CB is highly recommended during the idea exploration [10]. BWr combines individual and group conditions during the idea generation. Corroborating, a study showed that starting idea generation with individual condition alternate with group condition significantly enhance novel ideas [15]. For BWr, group members generate and share ideas written on paper or sticky notes, without talking, for 4 - 5 rounds of $8 \mathrm{~min} /$ round [15]. This method appears to provoke a significantly higher level of originality than does CB [22]. Nonetheless, this method shows disadvantage extent to which remaining fixed on certain ideas due to this method influencing participants dig deeply into specific categories of idea generated as time progresses [15,21].

Analogical thinking (AT) is another method which using analogy by gathering information/knowledge from previous innovative idea generation practices (the source) to generate new ones based on them (the target) [18,23]. Confirming, learners who have experienced or have similar problem to analogy can generate new ideas more effectively [23]. Advantageously, AT provides free time for combining knowledge which is named incubation. This step helps learners who are fixated on certain ideas to move forward [24]. Among these several methods used to promote idea generation, the hybrid approach is a new concept that has been getting consideration [25]. The hybrid approach refers to the combination of different cognitive stimulating methods; of different group interaction styles; or even of different external stimuli. The hybrid method helps in preventing loss of productivity [25] and emphasizes the innovative idea performance [15].

Previous study recommends a group-size of approximately 5 - 12 members to ensure effectiveness in brainstorming practice [26]. However, the most effective brainstorming practices consist of 6 participants [15]. Moreover, group-based brainstorming which consists of group members with diverse experiences and vary areas of expertise tends to generate significantly more novel ideas [27,28]. As seen, there are 2 general styles of group interactions for brainstorming sessions: a) face-to-face (verbal) interactions; and b) paper and pencil (non-verbal) interactions. The literature showed that the latter induces group members to express their ideas in writing [15,16,21] and tends to inhibit production blocking, evaluation apprehension, and social loafing/free riding to a greater extent than does face-to-face interactions [21]. This goes to show that providing proper external stimuli for brainstorming sessions can enhance the innovative ideas generation [17]; such stimuli consist of: 1) applying colorful sticky note papers and pens; 2) knowledge loading by providing useful information, or patents that is extracted from existing innovations during generating ideas [29,30]; and 3) building more ideas from viewing another person's ideas without talking [15]. Furthermore, nurses need skills to evaluate the performance or quality of the generated ideas: 1) fluency (the total number of non-redundant ideas); 2) flexibility (the number of different categories of ideas); and 3) originality (the unique or uncommon ideas) [17]. Thus, learning programs must provide idea performance appraisal activities and comprehensive idea evaluation tools that can enhance the manner relevant to idea generation.

The idea championing is the active promotion of a novel idea, aims at obtaining the approval to push the idea forward with achieving budget and time [31-33]. To promote this behavior, recent literature recommends organization providing opportunities for learners to sell the idea to organizational approver; "pitch meetings" [33]. Learners can enhance this behavior by practicing presentation with positive body language, highlight problem, clearly gaps indication of idea, idea goals, developing plan, and desired outcomes [34] to approver. Consequently, learners can perceive that their ideas have meaningful purpose [35]. Finally, idea implementation is a behavior in developing innovation and testing it. Workshop for turning innovative ideas into tangibles and testing their outcome increased sense of ownership and ability to produce innovation $[32,33,35]$. Organization can stimulate idea champion and idea implementation by investing in information sharing, supportive supervision, and establishing an innovative climate [36-38]. Likewise, organization climate to innovation affects employees' innovative work behavior [39]. Finally, IWB is stronger among nurses who frequently share their best practices and mistakes with co-workers [40]. 
Learning programs that provide a platform for interaction between persons and the environment to facilitate the transformation of people's experiences into new knowledge can cultivate the creativity of the learners $[4,41,44]$. Particularly, the experiential learning theory (ELT) illustrates a process of learning through experience, reflection on doing, conceptualization or thinking, and experimentation or hands-on $[42,43]$. Effectively, this theory increases group learning by promoting learners to participate in group activity. It dictates that learners' future behaviors can gain from their reflection throughout their experiences; meaning that persons can derive new knowledge from observation and experimentation. Corroborating, effective duration of learning which influences to IWB is at least 3 sessions within 2 days - 12 weeks $[44,45]$. Confirming, a study showed that group learning protocols significantly increase learner's creative capacity [46]. Nevertheless, learning programs to promote IWB and innovative idea performance among nurses are currently needing.

The MHB learning program is an experiential learning process over 12 weeks workshop. The program comprised training session, critical reflection and hands-on sessions with a set of leaning activities. The CB was applied for idea exploration. The MHB method, which was modified by combining the BWr, the AT and providing knowledge loading with the evidence-based practice (EBP), was used for enhancing idea generation manner. Idea pitching was an approach for promoting idea championing manner. Workshop and hands-on innovation development and testing were used to increase manner of idea implementation. Group-based of 6 participants with a wide range of experiences in nursing, providing instructions with appropriate length of session was assigned to enhance IWB. Thus, this study aimed to test the effectiveness of a modified hybrid brainstorming (MHB) learning program in enhancing IWB and fluency, flexibility, originality of its innovative idea performance among Thai nurses.

\section{Materials and methods}

\section{Research design and sample}

This study employed a 1 group pre-test and post-test design. It was conducted in January - April 2019. Study participants were nurses at a private hospital in Southern Thailand. Nurses who work at a private hospital in Southern Thailand (total staff $=372$ ) were chosen. The nurses who met the inclusion criteria; 1) working as nursing staff, 2) aged 25 - 55 years old, 3) with at least 3 years work experience, 4) normal or corrected-to-normal vision and 5) be able to fully participate until program completion, were approached and informed of the study. Two hundred and ten nurses were eligible. Lottery sampling by the nurse manager was performed to select 60 participants. Written informed consent was obtained before participating in the program. The sample size was estimated using power analysis with $\alpha=0.05$, power $=$ 0.80 and medium effect size $(\mathrm{d}=0.50)$ based on Cohen's table [47]; the sample size was calculated to be $50 ; 20 \%$ attrition was added to prevent the effects of participant drop-out. Thus, an estimate of 60 participants were required.

\section{Ethical consideration}

The study protocol was approved (Approval no. 2018 NSt - Qn 046) by the Center for Social and Behavioral Sciences Institutional Review Board, Prince of Songkla University, Thailand. All participants were given a brief explanation of study objectives, program activities, and were told that they were free to withdraw their participation at any time without receiving any penalty. First, participants provided verbal consent for participation; then, they were asked to fill out a written informed consent, which was obtained from all participants.

\section{Experimental intervention}

The MHB learning program (Figure 1) was developed as experimental intervention by the researcher based on the innovative work behavior aspects [6] and the ELT [43]. The learning program was a 12-week workshop long, comprising 3 sessions: 1) group training session; 2) group critical reflection session, and 3) hands-on session, with a set of learning activities (Figure 2). This learning program aimed at enhancing IWB and fluency, flexibility, originality of innovative idea performance. 


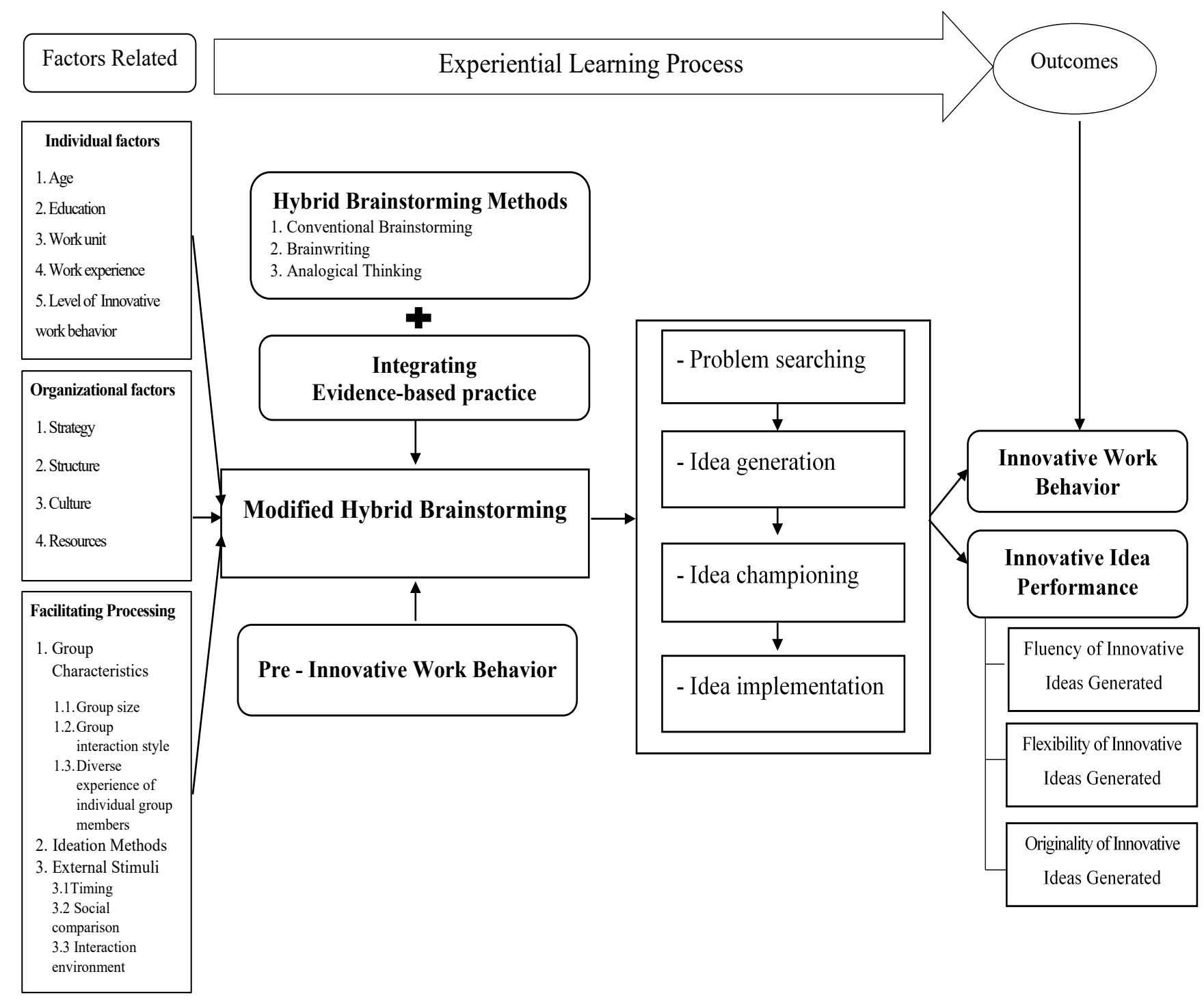

Figure 1 Conceptual framework of effectiveness of modified hybrid brainstorming learning program in increasing innovative work behavior and innovative idea performance among nurses. 


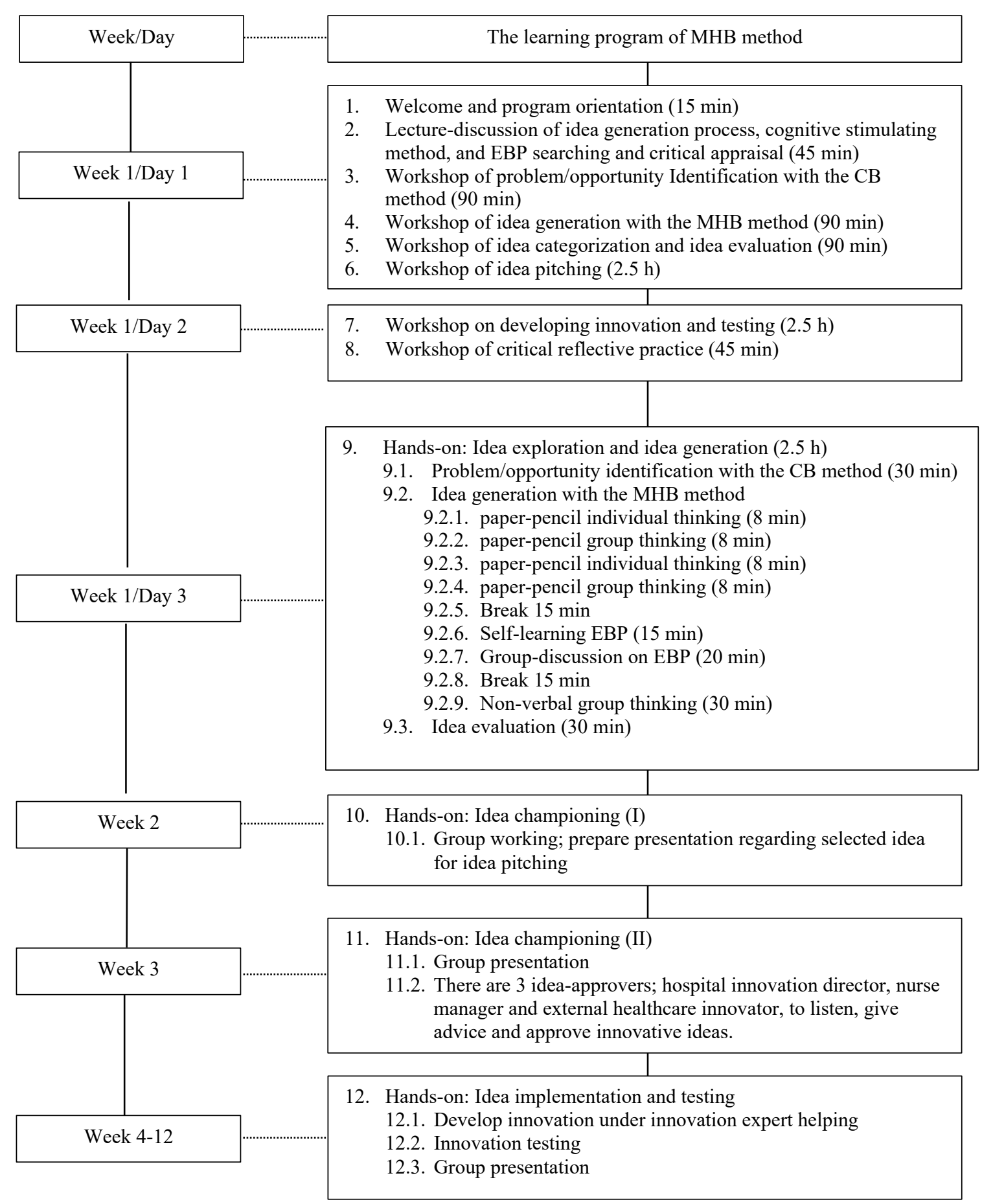

Figure 2 The intervention procedure of the study.

\section{Instruments for data collection}

IWB scores were obtained from the self-rated innovative work behavior questionnaire (SRIWBQ). The SRIWBQ was an existing tool used for measuring the degree of pre-innovative work behavior level which underwent a back translation process $[6,48]$. Reliability was established by administering the questionnaire to 30 nurses and Cronbach's alpha was 0.92 .

Innovative idea performance scale (IIPS) was used to evaluate fluency, flexibility, and originality of generated ideas. IIPS was adopted from Doungtongsuk's idea evaluation scale [49], which was modified based on Torrance's work [50]. Fluency refers to the total number of non-redundant ideas generated during a program. One non-redundant generated idea counted as a score of 1 . Flexibility is the number of 
different categories of generated ideas; 1 category counts as a score of 1 . Originality refers to the degree of uniqueness or uncommonness of generated ideas. Originality was measured by the infrequency of the idea based on the following: the generated idea will be assigned a statistical infrequency score of zero if $\geq$ $5 \%$ of the participants listed a given use, a score of 1 of $2-4.99 \%$ of the participants listed a given use and a score of 2 if $0-1.99 \%$ of the participants listed a given use. The percentage of absolute agreement for IIPS was $75-85 \%$.

Three experts in the area of innovation development were asked to assess content validity. One is a physician who works at the Faculty of Medicine, Prince of Songkla University, and an expert in medical innovation. The second expert is a lecturer from the Faculty of Business Economics and Communications, Naresuan University, who is an expert in innovation management. The third is an administrator nurse working in a private hospital who is an expert in nursing innovation. The index of item-objective congruence (IOC) was 0.89 .

\section{Data collection}

All participants were asked to take the self-rated IWB and generate innovative ideas regarding given condition on the first day before they started the learning program. At the hands-on of idea generation session, all participants were asked to generate innovative ideas. The researchers checked the ideas for completeness, coded the data and submitted all generated ideas to the experts for scoring fluency, flexibility and originality. At 12 weeks, the completion of the program, all participants were asked to retake the self-rated IWB questionnaire.

\section{Statistical analysis}

Descriptive statistics were used to analyze demographics, IWB, fluency, flexibility and originality of the generated ideas. A paired t-test was used to analyze the mean differences in IWB, fluency, flexibility and originality between baseline and post-test. The assumption test, the Shapiro-Wilk W test for normality testing was met.

\section{Results and discussion}

\section{Demographic characteristics}

Majority of the participants $(93.3 \%)$ were female nurses. The ages ranged from 26 to 44 years, with an average age of 33.6 years $(\mathrm{SD}=4.69)$. Almost all participants had attained a bachelor's degree $(93.3$ $\%)$ and had worked at an out- and in-patient service unit at the same rate $(50.0 \%)$. The majority of them worked as team member $(88.3 \%)$. The duration of nursing service experience ranged from 3 to 17 years, with a mean of 7.95 years $(\mathrm{SD}=3.87)$. Most of participants were not trained in brainstorming, problem solving, creativity skill, and innovation development. However, most of the participants had been trained in quality improvement (56.7\%) and quality tools (76.7\%) (Table 1).

Table 1 Characteristics of the participants.

\begin{tabular}{|c|c|c|c|}
\hline Characteristics & $\mathbf{n}$ & $\%$ & Mean \pm SD \\
\hline \multicolumn{4}{|l|}{ Gender: } \\
\hline Male & 4 & 6.7 & \\
\hline Female & 56 & 93.3 & \\
\hline Age (year): (min - max) $(26-44)$ & & & $33.60 \pm 4.69$ \\
\hline \multicolumn{4}{|l|}{ Education: } \\
\hline Bachelor's degree & 56 & 93.3 & \\
\hline Bachelor's degree with intensive course & 4 & 6.7 & \\
\hline \multicolumn{4}{|l|}{ Work location: } \\
\hline Out-patient service & 30 & 50.0 & \\
\hline In-patient service & 30 & 50.0 & \\
\hline \multicolumn{4}{|l|}{ Work position: } \\
\hline Team member & 53 & 88.3 & \\
\hline In-charge nurse & 7 & 11.7 & \\
\hline Work experience (year): (min - max) $(3-17)$ & & & $7.95 \pm 3.87$ \\
\hline
\end{tabular}




\begin{tabular}{lccc}
\hline \multicolumn{1}{c}{ Characteristics } & n & \% & Mean \pm SD \\
\hline Training experience in last 6 months & & & \\
Brainstorming: & 3 & 5.0 & \\
Yes & 57 & 95.0 & \\
No & & & \\
Problem solving: & 8 & 13.3 & \\
Yes & 52 & 86.7 \\
No & 2 & & \\
Creativity skill: & 58 & 96.3 \\
$\quad$ Yes & & \\
No & 9 & 15.0 \\
Innovation development: & 51 & 85.0 \\
Yes & 34 & 56.7 \\
No & 34 & 43.3 \\
Quality improvement: & 26 & \\
Yes & & \\
No & 46 & 23.7 \\
Quality tools: & 14 & \\
Yes & & \\
No &
\end{tabular}

\section{Effect of the MHB learning program on innovative work behavior}

The mean IWB scores at post-test (week 12) were higher than pre-test scores $(\mathrm{t}=-17.34, p<0.05)$ (Table 2). The mean scores of idea exploration $(\mathrm{M}=7.78, \mathrm{SD}=0.57)$, idea generation $(\mathrm{M}=10.50, \mathrm{SD}=$ $1.29)$, idea championing $(\mathrm{M}=6.78, \mathrm{SD}=0.89)$, and idea implementation $(\mathrm{M}=10.0, \mathrm{SD}=0.96)$ were also higher at post-test $(p<0.05)$ (Table 2). From these analyses, we can see that training using the MHB learning program increased IWB $(p<0.05)$.

Table 2 Comparison of the total scores and scores of different components of the innovative work behavior of nurses before and after intervention (12 weeks).

\begin{tabular}{cccccc}
\hline Manner & $\begin{array}{c}\text { Pre-test }(\mathbf{n}=\mathbf{6 0}) \\
\text { Mean } \pm \text { SD }(\mathbf{m i n}-\mathbf{m a x})\end{array}$ & $\begin{array}{c}\text { Post-test }(\mathbf{n}=\mathbf{6 0}) \\
\text { Mean } \pm \text { SD }(\mathbf{m i n}-\mathbf{m a x})\end{array}$ & $\mathbf{t}$ & $\boldsymbol{p}$ \\
\hline Idea exploration & $5.62 \pm 1.06(3-8)$ & $7.78 \pm 0.57(6-8)$ & -19.371 & $<0.05$ \\
Idea generation & $7.93 \pm 1.88(4-12)$ & $10.50 \pm 1.29(8-12)$ & -16.032 & $<0.05$ \\
Idea championing & $4.75 \pm 1.40(2-8)$ & $6.78 \pm 0.89(5-8)$ & -12.225 & $<0.05$ \\
Idea implementing & $8.02 \pm 2.15(2-12)$ & $10.0 \pm 0.96(9-12)$ & -8.451 & $<0.05$ \\
Total & $26.32 \pm 5.17(16-37)$ & $35.03 \pm 2.62(29-40)$ & -17.344 & $<0.05$ \\
\hline
\end{tabular}

The study showed that the implementation of the learning program with the MHB method increased all manners of innovative work behavior. The MHB learning program provided a welcoming atmosphere; the $1^{\text {st }}$ learning activity, for the participants which made them concerned of required knowledge, and the value of learning [53]. Consequently, this may have helped participants to perform the following learning activities and deal with the problems they confronted [53]. Later, the lecture-discussions were designed to help participants better understand which types of knowledge they should use during innovation development $[43,53]$. The $3^{\text {rd }}-7^{\text {th }}$ learning activities were designed to support them with experiences in all aspects of IWB: Idea exploration, idea generation, idea championing and idea implementation [43,53]. Group critical reflection, the $8^{\text {th }}$ learning activity, was designed to make participants consider their concrete experiences from various perspectives and articulate why and how they occurred [43]. Finally, the hands-on session: the $10^{\text {th }}$ through $12^{\text {nd }}$ learning activities, were meant to enhance their creativity and ability of innovation development from active experimentation $[43,44]$. The main reason for this was that the learning process was based on the experiential learning process that encouraged participants to 
increase critical, convergent and divergent thinking [4,41,43]. These results are consistent with Kolb [43], Hasan and colleagues [44] that learning program consisted of various activities and team-learning interaction through the process of gaining new knowledge or experiences, thinking, reflection, and handson could enrich IWB. Corroborating, learning practices with specific tools or method to each manner could increase creativity skill $[18,24,52]$ and self-ability for adult learners [35]. The finding is similar to previous studies which found that learning program comprised proving information sharing events, supportive supervision, and establishing an innovative climate $[36,37,38,54,55]$ could enhance nurses' IWB.

Effect of the MHB learning program on Fluency, flexibility, and originality of generated ideas

The mean fluency, flexibility and originality scores at post-test were higher than at pre-test (Table 3). Therefore, participants who were trained through the MHB learning program generated ideas with significantly higher fluency, flexibility and originality $(p<0.05)$.

Table 3 Comparison of fluency, flexibility, and originality pre- and post-training session.

\begin{tabular}{|c|c|c|c|c|c|c|}
\hline \multirow{3}{*}{ Innovative Idea Performance } & \multirow{2}{*}{\multicolumn{2}{|c|}{$\begin{array}{l}\text { Pre-Test } \\
(n=60)\end{array}$}} & \multirow{2}{*}{\multicolumn{2}{|c|}{$\begin{array}{c}\text { Post-Test } \\
(\mathrm{n}=60)\end{array}$}} & \multirow{3}{*}{$\mathbf{t}$} & \multirow{3}{*}{$p$} \\
\hline & & & & & & \\
\hline & Mean & $S D$ & Mean & $S D$ & & \\
\hline Fluency (Non-redundant idea) & 24.60 & 7.03 & 50.17 & 30.12 & -4.99 & $<0.05$ \\
\hline Flexibility (Categories of idea) & 11.9 & 6.95 & 22.50 & 9.46 & -4.73 & $<0.05$ \\
\hline Originality (Uniqueness of idea) & 105.0 & 21.46 & 224.2 & 26.20 & -14.37 & $<0.05$ \\
\hline
\end{tabular}

The participants who were trained with the MHB method grounded in the experiential learning process [43] generated statistically higher fluency, flexibility, and originality of ideas post-training session $(p<0.05)$. These findings are consistent with the tenet of the experiential learning theory [43], according to which a training process with combinations and associations from varying patterns of thought can produce diverse ideas. Moreover, learning through one's own experiences and interacting with one's own goals could increase knowledge leading to generation of new ideas. Moreover, the learning activities grounded in the concept of observing and reflecting could help participants generate new ideas [43].

Participants gained creative skills; divergent and convergent thinking, from practicing in groups of 6 individuals [15,26] with diverse characteristics [27,28], combined group interactions, released idea fixation by knowledge loading with related evidence-based practices [15,16,21], and providing free-time for incubation process before idea generation [18]. As in the hybrid brainstorming concept, the groupbased brainstorming method combined with different methods or conditions, allowing group members to share ideas without production blocking, social loafing, evaluation apprehension, and working with different team members characteristics under time restrictions could enhance innovative idea performance $[15,20,44,55]$.

As for group interaction style, the MHB method showed positive impact as participants generated more non-redundant, different of idea categories and original ideas by using effective group-based cognitive stimulation which combines individual and group-working in a free-flowing manner as in previous studies $[15,18,52]$. Other results with the MHB method that parallel previous reports were that knowledge loading with the evidence-based practice and taking time off for the unconscious mind to process and associate the information $[18,24]$ had a positive influence on participants regarding generating a higher number of original ideas. Moreover, participants trained with the MHB method who started idea generation with the individual condition first, alternated with group condition 4 - 5 rounds without talking interaction, generated more original ideas [15]. These results show the benefit of hybrid brainstorming method that participants who worked without distraction from others or lack of interference would produce more ideas [15,18,23-25]. Likewise, there was a statistically significant difference of flexibility mean scores between pre- and post-test $(\mathrm{t}=-4.73, p<0.05)$. The result of the pilot study by Radel et al. [55] had the same outcome, that is the participants who worked under less inhibition had 
more flexibility of ideas. This finding confirmed that group interaction style influenced flexibility of ideas generated $[15,16]$.

Therefore, our findings reveal that the MHB learning program improved participants' understanding of innovation process, promoted manner of idea exploration, idea generation, idea championing and implementation of ideas. Most importantly, it helps to increase the confidence of nurses in the step of innovative idea generation.

\section{Conclusions}

Our proposed MHB learning programs were able to enhance participants' IWB. Moreover, the MHB learning program provided greater enhancements toward idea fluency, flexibility, and originality after program completion. The study insists that nurses can enhance IWB and generate innovative ideas. Thus, organization should provide resources and facilitate nurses to behave as innovation champions and change agent.

\section{Acknowledgements}

The study was financially supported by the Graduate School, Prince of Songkla University, Thailand, by the grant for the funding of doctoral programs, under the Thailand's Education Hub for ASEAN Countries (TEH-AC) scholarship program and research dissertation.

\section{References}

[1] E Lopez, JL Gonzalez, JA Cordo, M Janvier-Anglade and TA Fitzpatrick. EntrepreNurses: Nursing's evolving role in innovation strategy. Nurs. Econ. 2019; 37, 159-63.

[2] T Kelley. Innovation in nursing. Am Nurse Assoc. Massachusetts 2019; 17, 1-12.

[3] D Carlucci, M Mura and G Schiuma. Fostering employees' innovative work behavior in healthcare organisations. Int. J. Innov. Manag. 2020; 24, 2050014.

[4] AH Awang, NM Sapie, MY Hussain, S Ishak and RM Yusof. Nurturing innovative employees: Effects of organisational learning and work environment. Econ. Res. 2019; 32, 1152-68.

[5] F Babiss, L Thomas and MM Fricke. Innovative team training for patient safety: Comparing classroom learning to experiential training. J. Contin. Educ. Nurse. 2017; 48, 563-9.

[6] JD Jong and DD Hartog. Innovative work behavior: Measurement and validation. EIM. Bus. Policy Res. 2008; 8, 1-27.

[7] I Hocking and D Vernon. The right tool for the right task: Structured techniques prove less effective on an ill-defined problem finding task. Think. Skills Creativ. 2017; 26, 84-91.

[8] J O'loghlin. Innovation is a state of mind. John Wiley \& Sons, Australia, 2016, p. 7-112.

[9] KL Foor. 2017, The significance of participation in an innovation training program on the perception of creative behavior. Ph.D. Dissertation. University of Phoenix, Southfield MI, United State.

[10] TR Koroh, P Setyosari, INS Degeng and IW Dasna. The effect of problem solving method vs brainstorming method and learning motivation towards learning outcomes in science. In: Proceedings of the $2^{\text {nd }}$ International Conference on Education, Malang, Indonesia. 2017, p.890-900.

[11] B Misiurek. Standardized work with TWI: Eliminating human errors in production and service processes. $1^{\text {st }}$ ed. Productivity Press, New York, 2016, p. 51-78.

[12] LJ Kornish and J Hutchison-Krupat. Research on idea generation and selection: Implications for management of technology. Prod. Oper. Manag. 2017; 26, 633-51.

[13] EF Rietzschel and BA Nijstad and W Stroebe. Effects of problem scope and creativity instructions on idea generation and selection. Creativ. Res. J. 2014; 2, 185-91.

[14] O Serrat. Harnessing creativity and innovation in the workplace. Knowledge solutions. Springer, Singapore, 2017, p. 903-10.

[15] R Korde and PB Paulus. Alternating individual and group idea generation: Finding the elusive synergy. J. Exp. Soc. Psychol. 2017; 70, 177-90.

[16] TM Russell. 2019, Interactive ideation: Online team-based idea generation versus traditional brainstorming. Ph.D. Dissertation. University of Minnesota, Minneapolis MN, United State.

[17] WI Hidayanti, D Rochintaniawati and RR Agustin. The effect of brainstorming on students' creative thinking skill in learning nutrition. J. Sci. Learn. 2018; 1, 44-8.

[18] E Kim. Workshop design for enhancing the appropriateness of idea generation using analogical thinking. Int. J. Innov. Stud. 2017; 1, 134-43. 
[19] H Al-Samarraie and S Hurmuzan. A review of brainstorming techniques in higher education. Think. Skills Creativ. 2018; 27, 78-91.

[20] H Besant. The journey of brainstorming. J. Transform. Innov. 2016; 2, 1-7.

[21] K Wang. Towards a taxonomy of idea generation techniques. Found. Manag. 2019; 11, 65-80.

[22] PB Paulus, RM Korde, JJ Dickson, A Carmeli and R Cohen-Meitar. Asynchronous brainstorming in an industrial setting: Exploratory studies. Hum. Factors 2015; 57, 1076-94.

[23] D Gentner and F Maravilla. Analogical reasoning. In: LJ Ball and VA Thompson (Eds.). International handbook of thinking \& reasoning. Routledge, New York, 2018, p. 186-203.

[24] HH Choi and MJ Kim. The effects of analogical and metaphorical reasoning on design thinking. Think. Skills Creativ. 2017; 23, 29-41.

[25] T Shealy, M Hu and J Gero. Patterns of cortical activation when using concept generation techniques of brainstorming, morphological analysis, and TRIZ. In: Proceedings of the ASME 2018 International Design Engineering Technical Conferences and Computers and Information in Engineering Conference, Quebec, Canada. 2018, p. 1-10.

[26] N Suzuki, M Imashiro, H Shoda, N Ito, M Sakata and M Yamamoto. Effects of group size on performance and member satisfaction. In: Proceedings of the $20^{\text {th }}$ International Conference on Human Interface and the Management of Information, Las Vegas NV, United State. 2018, p. 191-9.

[27] ME Haiba, L Elbassiti and R Ajhoun. Idea management: Idea generation stage with a qualitative focus. J. Adv. Manag. Sci. 2017; 5, 271-8.

[28] PB Paulus and JB Kenworthy. Effective brainstorming. In: PB Paulus and BA Nijstad (Eds.). Handbook of group creativity: Innovation through collaboration. Oxford University Press, New York, 2019, p. 287-386.

[29] G Cascini and N Becattini. A scientific framework for testing creativity enhancing techniques. In: Proceedings of the $28^{\text {th }}$ Annual Conference of the Australasian Association for Engineering Education, Sydney, Australia. 2017, p. 613-21.

[30] T Montag-Smit and CP Maertz. Searching outside the box in creative problem solving: The role of creative thinking skills and domain knowledge. J. Bus. Res. 2017; 81, 1-10.

[31] S Lu, KM Bartol, V Venkataramani, X Zheng and X Liu. Pitching novel ideas to the boss: The interactive effects of employees' idea enactment and influence tactics on creativity assessment and implementation. Acad. Manag. J. 2019; 62, 579-606.

[32] TW Thomas, PC Seifert and JC Joyner. Registered nurses leading innovative changes. Online J. Issues. Nurs. 2016; 21, p.1-15.

[33] JE Perry-Smith and PV Mannucci. Social networks, creativity, and entrepreneurship. In: CE Shalley, MA Hitt and J Zhou (Eds.). The oxford handbook of creativity, innovation, and entrepreneurship. Oxford University Press, New York, 2015, p. 205-24.

[34] M Kunte, T Promsiri and K Kampanthong. Components of entrepreneurial idea Pitch: A qualitative analysis from an Entrepreneurial classroom. J. Manag. Innov. 2018; 5, 107-17.

[35] C Zuber and L Moody. Creativity and innovation in healthcare: Tapping into organizational enablers through human centered design. Nurs. Adm. Quart. 2018; 42, 62-75.

[36] D Yan, F Wen, X Li and Y Zhang. The relationship between psychological capital and innovation behaviour in Chinese nurses. J. Nurs. Manag. 2020; 28, 471-9.

[37] S Nazir, W Qun, L Hui and A Shafi. Influence of social exchange relationships on affective commitment and innovative behavior: Role of perceived organizational support. Sustainability $2018 ; 10,4418$.

[38] AC Bos-Nehles and AA Veenendaal. Perceptions of HR practices and innovative work behavior: The moderating effect of an innovative climate. Int. J. Hum. Resour. Manag. 2019; 30, 2661-83.

[39] R Shanker, R Bhanugopan, BIVD Heijden and M Farrell. Organizational climate for innovation and organizational performance: The mediating effect of innovative work behavior. J. Vocat. Behav. 2017; 100, 67-77.

[40] B Afsar, S Cheema and BB Saeed. Do nurses display innovative work behavior when their values match with hospitals' values? Eur. J. Innov. Manag. 2018; 21, 157-71.

[41] EM Sutanto. The influence of organizational learning capability and organizational creativity on organizational innovation of Universities in East Java, Indonesia. Asia. Pac. Manag. Rev. 2017; 22, 128-35.

[42] DR Covington. 2018, The effects of ambidexterity training for team-level dynamic capabilities development. Ph.D. Dissertation. University of Trevecca Nazarene, Nashville TN, United State.

[43] DA Kolb. Experiential learning: Experience as the source of learning and development. $2^{\text {nd }}$ ed. Upper Saddle River, New Jersey, 2015, p. 31-61. 
[44] S Hasan and R Koning. Conversational peers and idea generation: Evidence from a field experiment. Harvard Business School, Stanford University Graduate School of Business, 2017.

[45] BE Watley. 2016, Effects of innovation training on innovative work behaviors. Ph.D. Dissertation. Regent University, Virginia Beach VA, United State.

[46] N Mayseless, M Saggar, G Hawthorne and A Reiss. Creativity in the twenty-first century: The added benefit of training and cooperation. In: $\mathrm{H}$ Plattner, C Meinel and L Leifer (Eds.). Design thinking research. Springer, Cham, 2018, p. 239-49.

[47] J Cohen. Statistical power analysis for the behavioral sciences. $2^{\text {nd }}$ ed. Hillsdale, New Jersey, 1988, p. 54.

[48] P Sawangwan. 2013, Head nurse - staff nurse exchange, structural empowerment and innovative behavior of professional nurses in regional hospitals. Master Thesis. Chaingmai University, Chaingmai, Thailand.

[49] P Doungtongsuk. 2017, Creativity development program based on holistic nervous system stimulation for administrators. Ph. D. Dissertation. Burapha University, Chon Buri, Thailand.

[50] EP Torrance. Research review for the torrance tests of creative thinking figural and verbal forms $A$ and B. Scholastic Testing Service, Bensenville IL, 2006.

[51] N Steils and S Hanine. Creative contests: Knowledge generation and underlying learning dynamics for idea generation. J. Mark. Manag. 2016; 32, 1647-69.

[52] J Sun, Q Chen, Q Zhang, Y Li, H Li, D Wei, Y Wenjing and Q Jiang. Training your brain to be more creative: Brain functional and structural changes induced by divergent thinking training. Hum. Brain Mapp. 2016; 10, 3375-87.

[53] MJ Bradshaw and AJ Lowenstein. Innovative teaching strategies in nursing and related health professions. $6^{\text {th }}$ ed. Jones \& Bartlett Learning, Massachusetts, 2014, p. 1-70.

[54] Tsai H, Liou S, Hsiao Y, Cheng C. The relationship of individual characteristics, perceived worksite support and perceived creativity to clinical nurses' innovative outcome. J. Clin. Nurs. 2013; 17-18, 2648-57.

[55] R Radel, K Davranche, M Fournier and A Dietrich. The role of (dis) inhibition in creativity: Decreased inhibition improves idea generation. Cognition 2015; 134, 110-20. 